\title{
Hall Effects on unsteady MHD flow of a Non-Newtonian fluid through a Porous medium with uniform suction and injection
}

\author{
${ }^{1}$ L.Sreekala*, ${ }^{2}$ E.Kesavareddy \\ 1) Assistant professor, Department of Mathematics, CRIT, Anantapur. \\ 2) Professor, Department of Mathematics, JNTU, Anantapur, A.P, India.
}

\begin{abstract}
In this Paper we discussed the unsteady magneto hydro dynamic flow of an electrically conducting viscous incompressible non-Newtonian Bingham fluid through a porous medium bounded by two parallel nonconducting porous plates with heat transfer considering the hall current effects into account. The fluid is driven by a uniform pressure gradient parallel to the channel plates and the entire flow field is subjected to a uniform magnetic field of strength $H_{o}$ with the normal to the boundaries in the transverse xz-plane. An external uniform magnetic field is applied perpendicular to the plates and the fluid motion is subjected to a uniform suction and injection. The lower plate is stationary and the upper plate moves with a constant velocity and the two plates are kept at different but constant temperatures. Numerical solutions are obtained for the governing momentum and energy equations taking the Joule and viscous dissipations into consideration. The effects of the Hall term, the parameter describing the non-Newtonian behaviour, and the velocity of suction and injection on both the velocity and temperature distributions is studied.
\end{abstract}

Keywords: Hall effects, unsteady flows, non-Newtonian flows, porous medium, Heat transfer, uniform suction and injection.

\section{Introduction}

The study of Couette flow in a rectangular channel of an electrically conducting viscous fluid under the action of a transversely applied magnetic field has immediate applications in many devices such as magneto hydro dynamic (MHD) power generators, MHD pumps, accelerators, aerodynamics heating, electrostatic precipitation, and polymer technology, and petroleum industry, purification of crude oil and fluid droplets sprays. Channel flows of a Newtonian fluid with heat transfer have been studied with or without Hall currents by many authors $[1,2,5-7,9,10-13,15,19$ and 20-24]. These results are important for the design of the duct wall and the cooling arrangements. The fluids that are used extensively in industrial applications are exhibiting a

yield stress $\tau_{0}$, that has to be exceeded before the fluid moves. As a result, such fluids cannot sustain a velocity gradient unless the magnitude of the local shear stress is higher than this yield stress. Fluids that belong to this category include cement, drilling mud, sludge, grease, granular suspensions, aqueous foams, slurries, paints, food products, plastics and paper pulp [24]. Due to the growing use of these non-Newtonian materials in various manufacturing and processing industries, considerable efforts have been directed towards understanding their flow and heat transfer characteristics. Many of the yield non-Newtonian fluids encountered in chemical engineering processes, are known to follow the so-called "Bingham model". A Bingham fluid is a material with a finite yield stress, followed by a linear curve at a finite strain rate. Many authors [4, 14, 16, 17 and 25] studied the flow or/and heat transfer of a Bingham fluid in different geometries. Attia [10] has studied the influence of the Hall current on the velocity and temperature fields of an unsteady Hartmann flow of a conducting Newtonian fluid between two infinite non-conducting horizontal parallel and porous plates. The extension of such problem to the case of Couette flow of non-Newtonian Bingham fluid is done in the present study. The upper plate is moving with a uniform velocity while the lower plate is stationary. The fluid is acted upon by a constant pressure gradient, a uniform suction from above, and a uniform injection from below and is subjected to a uniform magnetic field perpendicular to the plates. The Hall current is taken into consideration while the induced magnetic field is neglected by assuming a very small magnetic Reynolds number [21]. The two plates are kept at two different but constant temperatures. This configuration is a good approximation of some practical situations such as heat exchangers, flow meters, and pipes that connect system components. The Joule and viscous dissipations are taken into consideration in the energy equation. The governing momentum and energy equations are solved numerically using the finite difference approximations. The inclusion of the Hall current, the suction and injection, and the non-Newtonian fluid characteristics leads to some interesting effects on both the velocity and temperature fields. Recently, H.A.Attia [8] the unsteady magneto hydro dynamic flow of an electrically conducting viscous incompressible non-Newtonian Bingham fluid bounded by two parallel non-conducting porous plates is studied with heat transfer considering the Hall Effect. 


\section{Formulation and Solution of the problem:}

We consider an incompressible viscous and electrically conducting non Newtonian Bingham fluid in a parallel plate channel bounded by a loosely packed porous medium. The fluid is driven by a uniform pressure gradient parallel to the channel plates and the entire flow field is subjected to a uniform magnetic field of strength $\mathrm{H}_{\mathrm{o}}$ with the normal to the boundaries in the transverse xz-plane. The physical geometry of the problem is shown in Fig. 1. The fluid is assumed to be laminar, incompressible and obeying a Bingham model and flows between two infinite horizontal plates located at the $y= \pm h$ planes and extend from $\mathrm{x}=0$ to $\infty$ and from $\mathrm{z}=0$ to $\infty$. The upper plate moves with a uniform velocity $U_{0}$ while the lower plate is stationary. The upper and lower plates are kept at two constant temperatures $T_{2}$ and $T_{1}$ respectively, with $T_{2}>T_{1}$. The fluid is acted upon by a constant pressure gradient $\frac{d p}{d x}$ in the $\mathrm{x}$-direction, and a uniform suction from above and injection from below which are applied at $t=0$. A uniform magnetic field $\mathbf{B}_{0}$ is applied in the positive y-direction and is assumed undisturbed as the induced magnetic field is neglected by assuming a very small magnetic Reynolds number. The Hall Effect is taken into consideration and consequently a z-component for the velocity is expected to arise. The uniform suction implies that the y-component of the velocity is constant. Thus, the fluid velocity vector is given by

$$
v(y, t)=v(y, t) \mathbf{i}+v_{0} \mathbf{j}+w(y, t) \mathbf{k}
$$

It should be noted that the problem comes out to be a linear problem. In the hydrodynamic case without suction-injection, the problem reduces to Poiseuille problem [18] the classical hydro dynamic linear problem. Without suction-injection and by neglecting the Hall current, it reduces to Hartmann-Poiseuille problem [21]; the classical MHD linear problem. The inclusion of the constant suction-injection as well as the Hall term [10] preserves linearity. So obviously does changing the Newtonian fluid to a non-Newtonian one in the present study. The classical problems (Poiseuille and Hartmann-Poiseuille) of channel flow and the related pipe flow of Newtonian fluid are known to be attainable in practice and to give results in excellent agreement with experiments. The fully developed profiles are observed away from the inlet and the side-walls of the channel. Using a non-Newtonian fluid is not expected to cause a problem.

The fluid motion starts from rest at $\mathrm{t}=0$, and the no-slip condition at the plates implies that the fluid velocity has neither a $\mathrm{z}$ nor an $\mathrm{x}$-component at $y= \pm h$. The initial temperature of the fluid is assumed to be equal to $T_{1}$. Since the plates are infinite in the $\mathrm{x}$ and $\mathrm{z}$-directions, the physical quantities do not change in these directions.

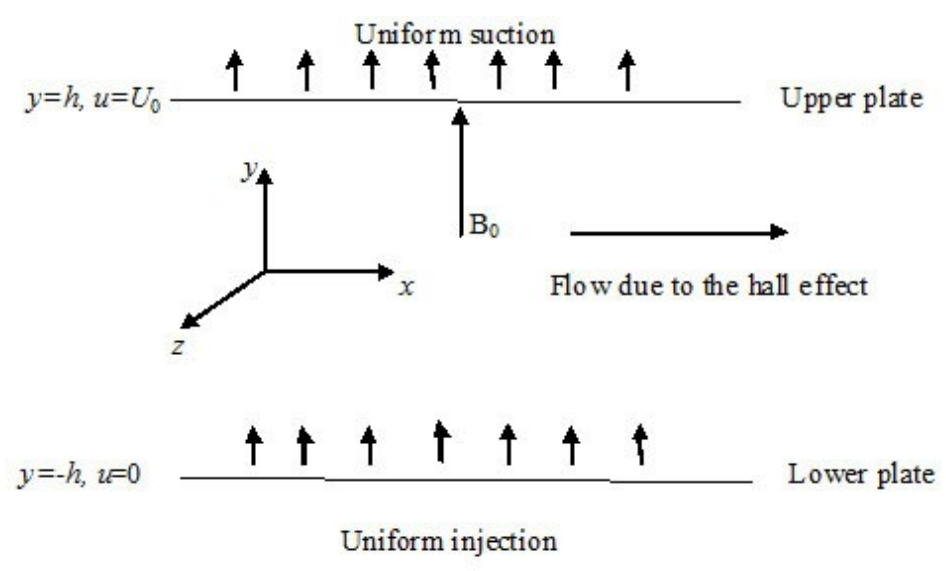

Fig. 1: The Physical configuration of the problem

The flow of the fluid is governed by the momentum equation

$\rho \frac{D v}{D t}=\nabla \cdot(\mu \nabla v)-\nabla p+\mathbf{J} \times \mathbf{B}_{0}$

where $\rho$ is the density of the fluid and $\mu$ is the apparent viscosity of the model and is given by

$$
\mu=K+\frac{\tau_{0}}{\sqrt{\left(\frac{\partial u}{\partial y}\right)^{2}+\left(\frac{\partial w}{\partial y}\right)^{2}}},
$$


where $\mathrm{K}$ is the plastic viscosity of a Bingham fluid, $\tau_{0}$ is the yield stress. If the Hall term is retained, the current density $\mathbf{J}$ is given by

$\mathbf{J}=\sigma\left[\mathbf{V} \times \mathbf{B}_{0}-\beta\left(\mathbf{J} \times \mathbf{B}_{0}\right)\right]$

where $\sigma$ is the electric conductivity of the fluid and $\beta$ is the Hall factor [21]. The equation (4.2.3) may be solved in $\mathbf{J}$ to yield

$$
\mathrm{J} \times \mathrm{B}_{0}=-\frac{\sigma B_{0}^{2}}{1+m^{2}}[(u+m w) \mathrm{i}+(w-m u) \mathrm{k}],
$$

where $\mathrm{m}$ is the Hall parameter and $m=\sigma \beta B_{0}$.

Thus, the two components of the momentum equation (4.2.1) becomes

$$
\begin{aligned}
& \rho \frac{\partial u}{\partial t}+\rho v_{0} \frac{\partial u}{\partial y}=-\frac{d p}{d x}+\frac{\partial}{\partial y}\left(\mu \frac{\partial u}{\partial y}\right)-\frac{\sigma B_{0}^{2}}{1+m^{2}}(u+m w)-\frac{v}{k} u \\
& \rho \frac{\partial w}{\partial t}+\rho v_{0} \frac{\partial w}{\partial y}=\frac{\partial}{\partial y}\left(\mu \frac{\partial u}{\partial y}\right)-\frac{\sigma B_{0}^{2}}{1+m^{2}}(w-m u)-\frac{v}{k} w
\end{aligned}
$$

The energy equation with viscous and Joule dissipations is given by

$$
\rho c_{p} \frac{\partial T}{\partial t}+\rho c_{p} v_{0} \frac{\partial T}{\partial y}=k \frac{\partial^{2} T}{\partial y^{2}}+\mu\left[\left(\frac{\partial u}{\partial y}\right)^{2}+\left(\frac{\partial w}{\partial y}\right)^{2}\right]+\left(\frac{\sigma B_{0}^{2}}{1+m^{2}}-\frac{v}{k}\right)\left(u^{2}+w^{2}\right)
$$

Where $c_{p}$ and $\mathrm{k}$ are, respectively, the specific heat capacity and the thermal conductivity of the fluid. The second and third terms on the right-hand side represent the viscous and Joule dissipations respectively. We notice that each of these terms has two components. This is because the hall effect brings about a velocity $\mathrm{w}$ in the z-direction. The initial and boundary conditions of the problem are given by

$u=w=0$ at $t \leq 0$,

$w=0$ at $\mathrm{y}=-\mathrm{h}$ and $\mathrm{y}=\mathrm{h}$ for $t>0$

$\mathrm{u}=0$ at $\mathrm{y}=-\mathrm{h}$ for $\mathrm{t}>0, \mathrm{u}=\mathrm{U}_{0}$ at $\mathrm{y}=\mathrm{h}$ for $\mathrm{t}>0$

$\mathrm{T}=\mathrm{T}_{1}$ at $t \leq 0$,

$\mathrm{T}=\mathrm{T}_{1}$ at $\mathrm{y}=\mathrm{h}, \mathrm{T}=\mathrm{T}_{2}$ at $\mathrm{y}=-\mathrm{h}$ for $\mathrm{t}>0$

That the boundary conditions do not show dependence on $\mathrm{x}$ suggests that the problem has a fully developed solution of the form, $\mathrm{u}=\mathrm{u}(\mathrm{y}, \mathrm{t}), \mathrm{v}=\mathrm{v}_{0}, \mathrm{p}=\mathrm{P}+\mathrm{Gx}$

where $\mathrm{P}$ is the pressure at $\mathrm{x}=0$ (constant), $\mathrm{G}$ is the constant pressure gradient (negative). Under these conditions the continuity equation $\frac{\partial u}{\partial x}+\frac{\partial v}{\partial y}=0$ is automatically satisfied. It is expedient to write the above equations in the non-dimensional form. To do this, we introduce the following non-dimensional quantities

$$
x^{*}=\frac{x}{h}, y^{*}=\frac{y}{h}, z^{*}=\frac{z}{h}, t^{*}=\frac{t U_{0}}{h}, w^{*}=\frac{w}{U_{0}}, p^{*}=\frac{p}{\rho U_{0}^{2}}, \theta=\frac{T-T_{1}}{T_{2}-T_{1}}, \mu^{*}=\frac{\mu}{K}
$$

Making use of the above non-dimensional variables, the equations from (5) to (12) and (2) are, respectively, written as (where the asterisks are dropped for convenience)

$$
\begin{aligned}
& \frac{\partial u}{\partial t}+\frac{S}{\operatorname{Re}} \frac{\partial u}{\partial y}=-\frac{d p}{d x}+\frac{1}{\operatorname{Re}}\left[\frac{\partial}{\partial y}\left(\mu \frac{\partial u}{\partial y}\right)-\frac{M^{2}}{1+m^{2}}(u+m w)-\frac{v}{k} u\right], \\
& \frac{\partial w}{\partial t}+\frac{S}{\operatorname{Re}} \frac{\partial w}{\partial y}=-\frac{d p}{d x}+\frac{1}{\operatorname{Re}}\left[\frac{\partial}{\partial y}\left(\mu \frac{\partial w}{\partial y}\right)-\frac{M^{2}}{1+m^{2}}(w-m u)-\frac{v}{k} w\right], \\
& \frac{\partial \theta}{\partial t}+\frac{S}{\operatorname{Re}} \frac{\partial \theta}{\partial y}=\frac{1}{\operatorname{Pr}} \frac{\partial^{2} T}{\partial y^{2}}+E c \mu\left[\left(\frac{\partial u}{\partial y}\right)^{2}+\left(\frac{\partial w}{\partial y}\right)^{2}\right]+\left(\frac{\sigma B_{0}^{2}}{1+m^{2}}-\frac{v}{k}\right)\left(u^{2}+w^{2}\right)
\end{aligned}
$$

Corresponding the initial and boundary conditions are

$$
\begin{aligned}
& u=w=0 \text { at } t \leq 0, \\
& u=w=0 \text { at } \mathrm{y}=-1
\end{aligned}
$$


$\mathrm{u}=1, \mathrm{w}=0$ at $\mathrm{y}=1$ for $\mathrm{t}>0$,

$\theta=0$ for $t \leq 0$, and $\theta=0$ at $\mathrm{y}=-1, \theta=1$ at $\mathrm{y}=1$ for $\mathrm{t}>0$

$\mu=1+\frac{\tau_{D}}{\sqrt{\left(\frac{\partial u}{\partial y}\right)^{2}+\left(\frac{\partial w}{\partial y}\right)^{2}}}$,

Where

$\tau_{D}=\frac{\tau_{0} h}{K U_{0}}$ is the Bingham number (dimensionless yield stress);

$R e=\frac{\rho U_{0} h}{K}$ is the Reynolds number;

$S=\frac{\rho v_{0} h}{K}$ is the suction parameter;

$\operatorname{Pr}=\frac{\rho c_{p} U_{0} h}{K}$ is the Prandtl number;

$E c=\frac{U_{0} K}{\rho c_{p} h\left(T_{2}-T_{1}\right)}$ is the Eckert number;

$M^{2}=\frac{\sigma B_{0}^{2} h^{2}}{K}$ is the Hartmann number squared:

$D^{-1}=\frac{h^{2}}{k K}$ is the inverse Darcy parameter

Eqs. (13), (14) and (20) represent coupled system of non-linear partial differential equations which are solved numerically under the initial and boundary conditions $(16,17$ and 18) using the finite difference approximations. The Crank-Nicolson implicit method is used [3]. Finite Difference equations relating the variables are obtained by writing the equations at the midpoint of the computational cell and then replacing the different terms by their second order central difference approximations in the y-direction. The diffusion terms are replaced by the average of the central differences at two successive time-levels. In equations (13) and (14) the non-linear terms are first linearized and then an iterative scheme is used at every time step to solve the linearized system of difference equations using Thomas algorithm to determine the velocity distributions. The values of the velocity components are substituted in the right-hand side of equation (15) which is solved numerically under the initial and boundary conditions (19). The computational domain is divided into meshes each is of dimension $\Delta t$ and $\Delta y$ in time and space respectively as shown in Fig. 2.

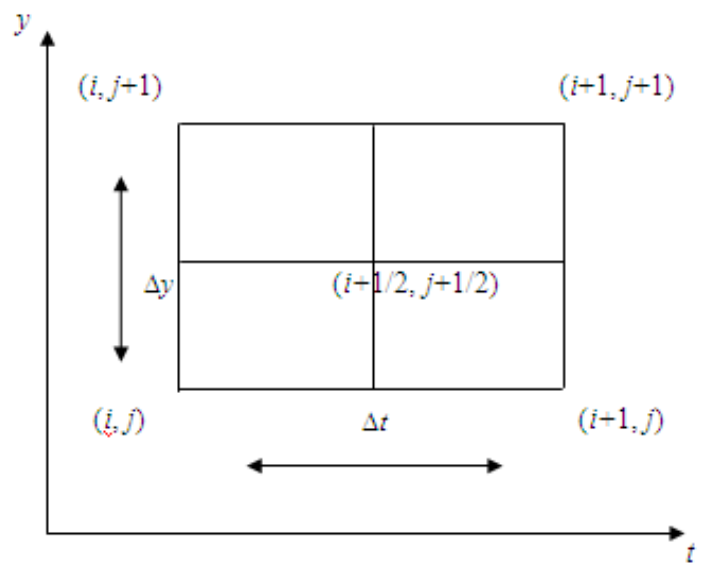

Fig. 2. Mesh diagram 
We define the variables $v=\frac{\partial u}{\partial y}, B=\frac{\partial w}{\partial y}, H=\frac{\partial \theta}{\partial y}$ and $\mu^{\prime}=\frac{\partial \mu}{\partial y}$ to reduce the second order differential equations to first order differential equations. The finite difference representations for equations (.13) and (14) take the form

$$
\begin{aligned}
& \left(\frac{u_{i+1, j+1}-u_{i, j+1}+u_{i+1, j}-u_{i, j}}{2 \Delta t}\right)+\frac{S}{\operatorname{Re}}\left(\frac{v_{i+1, j+1}+v_{i, j+1}+v_{i+1, j}+v_{i, j}}{4}\right)= \\
& -\frac{d p}{d x}+\left(\frac{\bar{\mu}_{i, j+1}+\bar{\mu}_{i, j}}{2 \operatorname{Re}}\right)\left(\frac{v_{i+1, j+1}+v_{i, j+1}-v_{i+1, j}-v_{i, j}}{2 \Delta y}\right)+ \\
& \left(\frac{\bar{\mu}_{i, j+1}^{\prime}+\bar{\mu}_{i, j}^{\prime}}{2 \operatorname{Re}}\right)\left(\frac{v_{i+1, j+1}+v_{i, j+1}-v_{i+1, j}-v_{i, j}}{2 \Delta y}\right) \\
& -\left(\frac{M^{2}}{1+m^{2}}+D^{-1}\right)\left(\frac{u_{i+1, j+1}+u_{i, j+1}+u_{i+1, j}+u_{i, j}}{2 \Delta t}+m \frac{w_{i+1, j+1}+w_{i, j+1}+w_{i+1, j}+w_{i, j}}{2 \Delta t}\right) \\
& \left(\frac{w_{i+1, j+1}-w_{i, j+1}+w_{i+1, j}-w_{i, j}}{2 \Delta t}\right)+\frac{S}{\operatorname{Re}}\left(\frac{B_{i+1, j+1}+B_{i, j+1}+B_{i+1, j}+B_{i, j}}{4}\right)= \\
& \left(\frac{\bar{\mu}_{i, j+1}+\bar{\mu}_{i, j}}{2 \operatorname{Re}}\right)\left(\frac{B_{i+1, j+1}+B_{i, j+1}-B_{i+1, j}-B_{i, j}}{4}\right)\left(\frac{B_{i+1, j+1}+B_{i, j+1}+B_{i+1, j}+B_{i, j}}{4 R e}\right)- \\
& -\left(\frac{M^{2}}{1+m^{2}}+D^{-1}\right)\left(\frac{w_{i+1, j+1}+w_{i, j+1}+w_{i+1, j}+w_{i, j}}{4 \operatorname{Re}}-m \frac{u_{i+1, j+1}+u_{i, j+1}+u_{i+1, j}+u_{i, j}}{4 R e}\right)
\end{aligned}
$$

The variables with bars are given initial guesses from the previous time steps and an iterative scheme is used at every time to solve the linearized system of difference equations. Then the finite difference form for the energy equation (4.2.15) can be written as

$$
\begin{aligned}
& \left(\frac{\theta_{i+1, j+1}-\theta_{i, j+1}+\theta_{i+1, j}-\theta_{i, j}}{2 \Delta t}\right)+\frac{S}{R e}\left(\frac{H_{i+1, j+1}+H_{i, j+1}+H_{i+1, j}+H_{i, j}}{4}\right)= \\
& =\frac{1}{\operatorname{Pr}}\left(\frac{H_{i+1, j+1}+H_{i, j+1}-H_{i+1, j}-H_{i, j}}{2 \Delta t}\right)+D I S P
\end{aligned}
$$

Where, DISP represents the Joule and viscous dissipation terms which are known from the solution of the momentum equations and can be evaluated at the midpoint $(\mathrm{i}, \mathrm{j})$ of the computational cell. Computations have been made for $(\mathrm{dp} / \mathrm{dx})=5, \operatorname{Pr}=1, \operatorname{Re}=1, \alpha=\pi / 3$ and $\mathrm{Ec}=0.2$. Step sides $\Delta t=0.0001$ and $\Delta y=0.005$ for time and space respectively, are chosen and the scheme converges in almost 7 iterations at every time step. Smaller step sizes do not show any significant change in the results. Convergence of the scheme is assumed when every one of $\mathrm{u}, \mathrm{v}, \mathrm{w}, \mathrm{B}, \theta$ and $\mathrm{H}$ for the last two approximations differ from unity by less than $10^{-6}$ for all values of $\mathrm{y}$ in $-1<\mathrm{y}<1$ at every time step. Less than seven approximations are required to satisfy this convergence criteria for all ranges of the parameters studied here. In order to examine the accuracy and correctness of the solutions, the results of the time development of the velocity components $u$ and $w$ at the centre of the channel for the Newtonian case is compared and shown, as depicted Table 1, to have complete agreement with those reported by Attia [10]. This ensures the satisfaction of all the governing equations; mass continuity, momentum and energy equations. 
Hall Effects on unsteady MHD flow of a Non-Newtonian fluid through a Porous medium with .....

\begin{tabular}{|c|c|c|c|c|c|c|}
\hline \multirow{2}{*}{$\mathrm{t}$} & \multicolumn{3}{|c|}{ The values of $\mathrm{u}$} & \multicolumn{3}{c|}{ The values of $\mathrm{w}$} \\
\cline { 2 - 7 } & $\begin{array}{c}\text { Present } \\
\text { Results }\end{array}$ & Attia [8] & Attia [10] & $\begin{array}{c}\text { Present } \\
\text { Results }\end{array}$ & Attia [8] & Attia [10] \\
\hline 0.1 & 0.5448 & 0.4673 & 0.4669 & 0.0445 & 0.0603 & 0.0619 \\
\hline 0.2 & 0.6326 & 0.8094 & 0.8089 & 0.0842 & 0.2060 & 0.2056 \\
\hline 0.3 & 0.6815 & 1.0165 & 1.0160 & 0.0912 & 0.3692 & 0.3687 \\
\hline 0.4 & 0.7968 & 1.1254 & 1.1251 & 0.1321 & 0.5177 & 0.5171 \\
\hline 0.5 & 0.8411 & 1.1709 & 1.1708 & 0.5201 & 0.6375 & 0.6370 \\
\hline 0.6 & 0.8665 & 1.1791 & 1.1791 & 0.6642 & 0.7264 & 0.7260 \\
\hline 0.7 & 0.8925 & 1.1681 & 1.1682 & 0.7745 & 0.7876 & 0.7872 \\
\hline 0.8 & 0.9962 & 1.1494 & 1.1495 & 0.7902 & 0.8266 & 0.8263 \\
\hline 0.9 & 1.0000 & 1.1295 & 1.1297 & 0.8326 & 0.8466 & 0.8491 \\
\hline 1.0 & 1.0001 & 1.1120 & 1.1122 & 0.8523 & 0.8693 & 0.8607 \\
\hline
\end{tabular}

Table 1: Comparison of the present results and the known results of Attia [8] and Attia [10] for Newtonian fluid $\tau_{D}=0$ for $\mathrm{M}=2, \alpha=\pi / 2, \mathrm{~m}=1, \mathrm{~S}=1$ and $\mathrm{y}=0$.

\section{Graphs and Tables:}
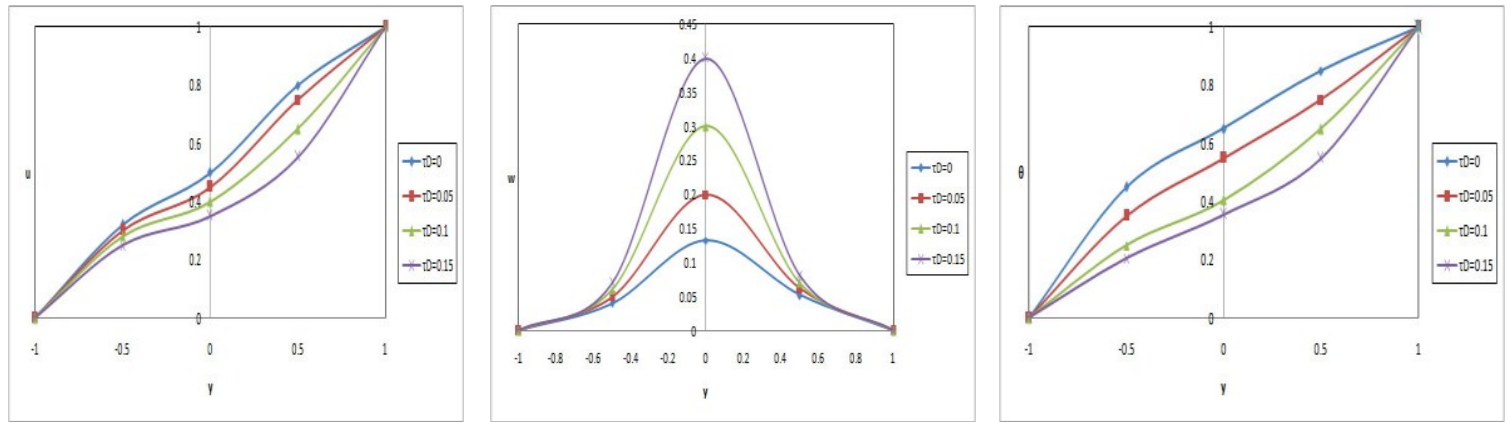

Fig 1 \& 2: The velocity profile for $u$, w and Temperature $\theta$ against $\tau_{D}$ with $t=0.1, \mathrm{~S}=1, \mathrm{M}=2, \mathrm{D}^{-1}=1000, m=1$
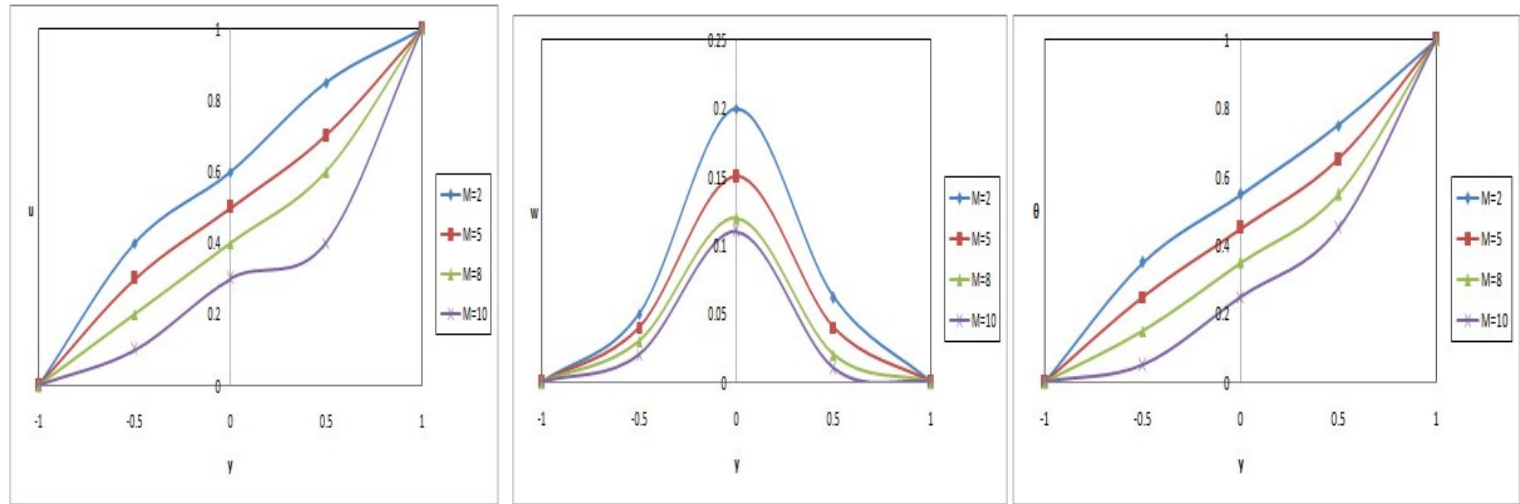

Fig 4, 5 \&6: The velocity profile for $u, w$ and Temperature $\theta$ against $\mathrm{M}$ with $t=0.1, \mathrm{~S}=1, \tau_{D}=0.05, \mathrm{D}^{-1}=1000, m=1$
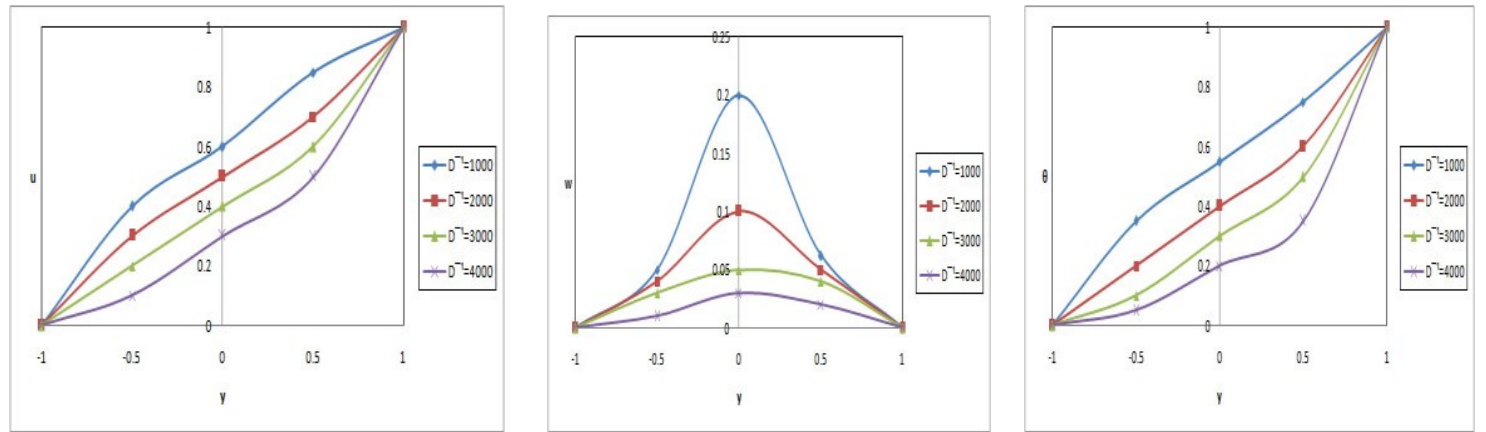

Fig 7,8\&9: The velocity profile for $u, w$ and Temperature $\theta$ against $\mathrm{D}^{-1}$ with $t=0.1, \mathrm{~S}=1, \tau_{D}=0.05, \mathrm{M}=2, m=1$ 

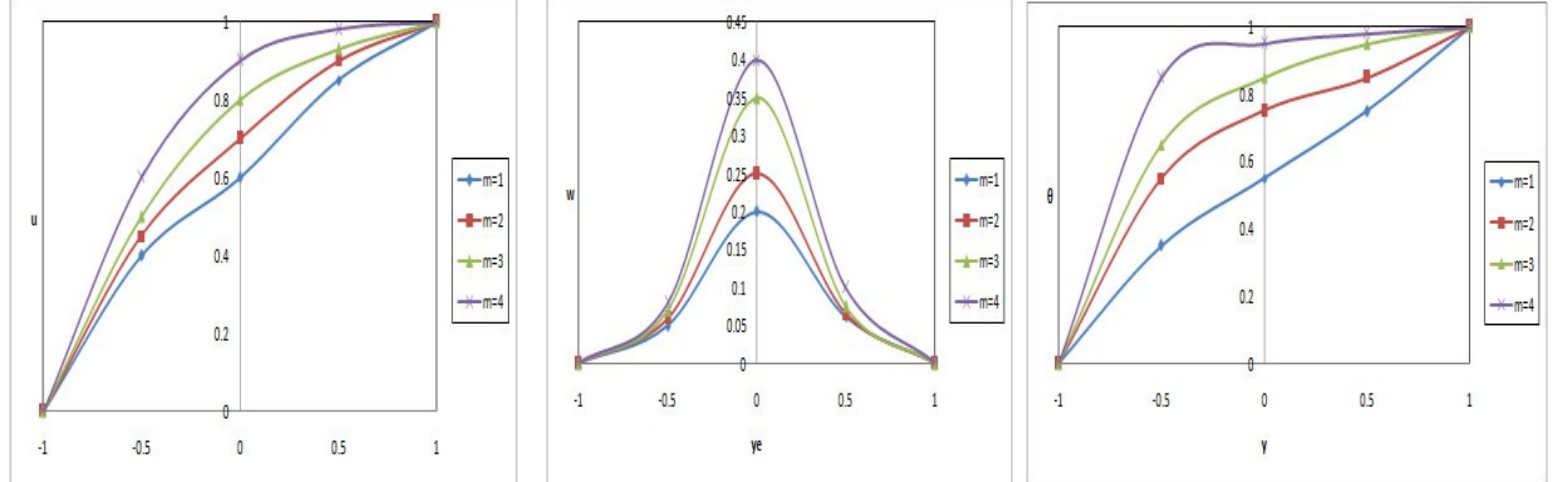

Fig 10,11\&12: The velocity profile for $u, w$ and Temperature $\theta$ against $m$ with $t=0.1, \mathrm{~S}=1, \mathrm{M}=2, \tau_{D}=0.05, \mathrm{D}^{-1}=1000$

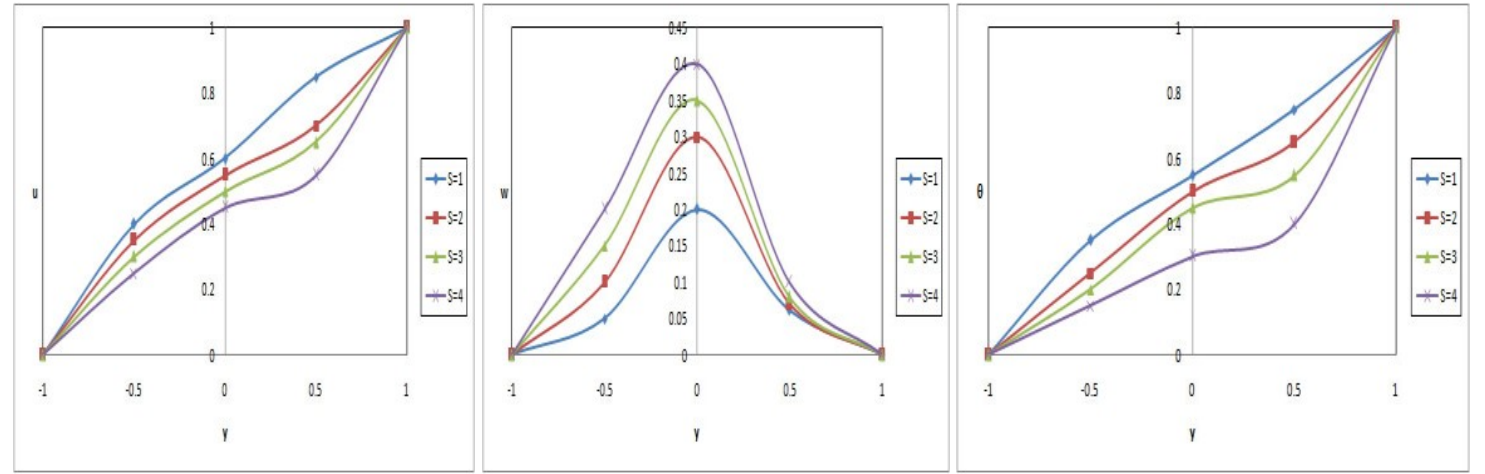

Fig 13,14\&15: The velocity profile for $u, w$ and Temperature $\theta$ against $\mathrm{S}$ with $t=0.1, m=1, \mathrm{M}=2, \tau_{D}=0.05, \mathrm{D}^{-1}=1000$
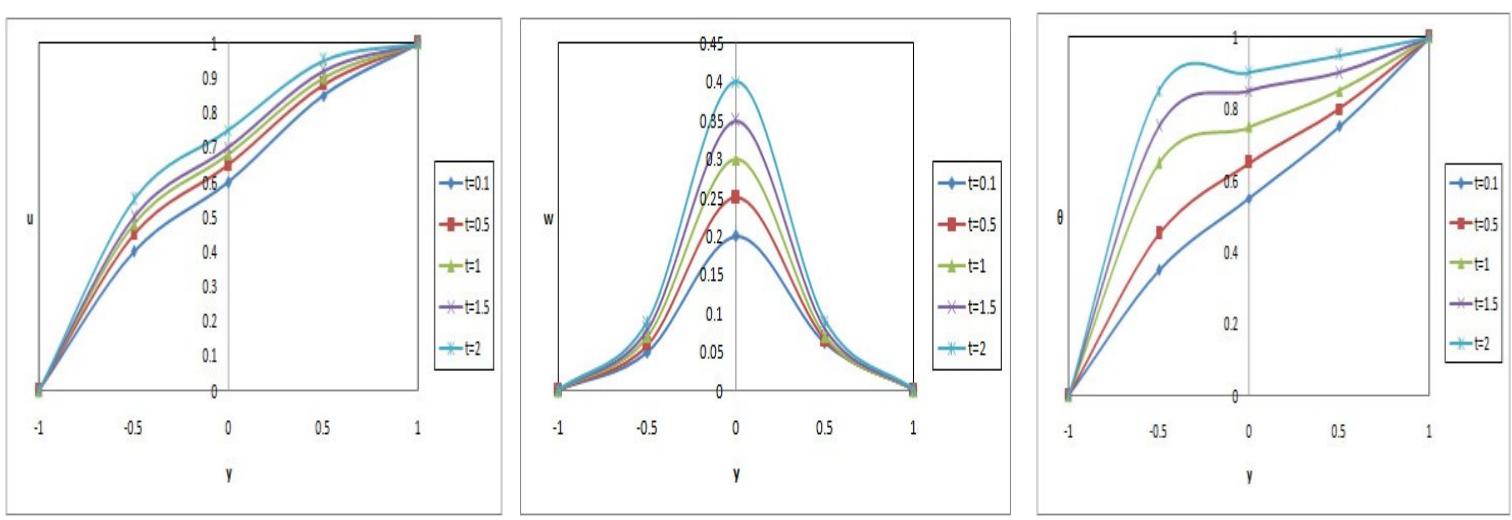

Fig 16, 17 \& 18: Time development of the velocity component $u, v \& \theta$ for $m=1, M=2, \tau_{D}=0.05, D^{-1}=1000, S=1$

\begin{tabular}{|c|c|c|c|c|c|c|c|c|c|}
\hline$\tau_{D}$ & I & II & III & IV & V & VI & VII & VIII & IX \\
\hline 0.05 & 0.4323 & 0.5143 & 0.5574 & 0.8450 & 0.9310 & 1.1531 & 2.4567 & 0.0521 & 0.0243 \\
\hline 0.1 & 0.4531 & 0.5312 & 0.5983 & 0.9312 & 1.0831 & 1.3342 & 3.4531 & 0.0632 & 0.0752 \\
\hline 0.15 & 0.4874 & 0.5575 & 0.6334 & 1.5244 & 1.6350 & 2.3423 & 4.4524 & 0.0834 & 0.0963 \\
\hline 0.2 & 0.5210 & 0.5896 & 0.6987 & 1.6250 & 1.8554 & 2.5542 & 5.0023 & 0.0986 & 0.1012 \\
\hline
\end{tabular}

\begin{tabular}{|c|c|c|c|c|c|c|c|c|c|}
\hline & I & II & III & IV & V & VI & VII & VIII & IX \\
\hline $\mathrm{M}$ & 2 & 5 & 8 & 2 & 2 & 2 & 2 & 2 & 2 \\
\hline $\mathrm{D}^{-1}$ & 1000 & 1000 & 1000 & 2000 & 3000 & 1000 & 1000 & 1000 & 1000 \\
\hline $\mathrm{m}$ & 1 & 1 & 1 & 1 & 1 & 2 & 3 & 1 & 1 \\
\hline $\mathrm{S}$ & 1 & 1 & 1 & 1 & 1 & 1 & 1 & 2 & 3 \\
\hline
\end{tabular}

Table. 1: The Nusselt number $(\mathrm{Nu})$ at $\mathrm{y}=1$ level 
Hall Effects on unsteady MHD flow of a Non-Newtonian fluid through a Porous medium with .....

\begin{tabular}{|c|c|c|c|c|c|c|c|c|c|}
\hline$\tau_{D}$ & I & II & III & IV & V & VI & VII & VIII & IX \\
\hline 0.05 & 0.0312 & 0.0402 & 0.0518 & 0.1452 & 0.2844 & 0.3562 & 0.5223 & 0.0052 & 0.0043 \\
\hline 0.1 & 0.0542 & 0.0631 & 0.0754 & 0.2564 & 0.4312 & 0.4872 & 0.6556 & 0.0072 & 0.0052 \\
\hline 0.15 & 0.0630 & 0.0743 & 0.0831 & 0.3784 & 0.5755 & 0.6674 & 0.8596 & 0.0084 & 0.0071 \\
\hline 0.2 & 0.0751 & 0.0841 & 0.0986 & 0.4007 & 0.6633 & 0.7884 & 1.0024 & 0.0096 & 0.0083 \\
\hline
\end{tabular}

\begin{tabular}{|c|c|c|c|c|c|c|c|c|c|}
\hline & I & II & III & IV & V & VI & VII & VIII & IX \\
\hline $\mathrm{M}$ & 2 & 5 & 8 & 2 & 2 & 2 & 2 & 2 & 2 \\
\hline $\mathrm{D}^{-1}$ & 1000 & 1000 & 1000 & 2000 & 3000 & 1000 & 1000 & 1000 & 1000 \\
\hline $\mathrm{m}$ & 1 & 1 & 1 & 1 & 1 & 2 & 3 & 1 & 1 \\
\hline $\mathrm{S}$ & 1 & 1 & 1 & 1 & 1 & 1 & 1 & 2 & 3 \\
\hline
\end{tabular}

Table. 2: The Nusselt number $(\mathrm{Nu})$ at $\mathrm{y}=-1$ level

\section{Results and Discussion:}

The flow governed by the non-dimensional parameters $\tau_{D}$ Bingham number, Re Reynolds number, $\mathrm{M}$ Hartmann number, $\mathrm{D}^{-1}$ inverse Darcy parameter, Pr Prandtl number, Ec the Eckert number and $\mathrm{S}$ suction parameter. Figs. (1-18) present the profiles of the velocity components $\mathrm{u}$ and $\mathrm{w}$ and the temperature $\theta$ respectively for fixed value of time $\mathrm{t}$ and for $\tau_{D}=0,0.05$ and 0.1 . The figures are evaluated for $\mathrm{M}=2, \mathrm{~m}=1$, and $\mathrm{S}=1$. It is clear from figures (1-3) that increasing the yield stress $\tau_{D}$ decrease the velocity components $\mathrm{u}$ and increase the velocity component $\mathrm{w}$ and the time at which they reach their steady state values as a result of increasing the viscosity. The figures show also that the velocity components $u$ and $w$ do not reach their steady state monotonically. This behaviour is more pronounced for small values of the parameter $\tau_{D}$ and it is clearer for $\mathrm{u}$ than for $\mathrm{w}$. Figure (3) shows that the temperature profile reaches its steady state monotonically. It is observed also that the velocity component $\mathrm{u}$ reaches the steady state faster than $\mathrm{w}$ which, in turn, reaches the steady state faster than $\theta$. This is expected as $\mathrm{u}$ is the source of $\mathrm{w}$, while both $\mathrm{u}$ and $\mathrm{w}$ act as sources for the temperature. Figures (4-6) depict the variation of the velocity components $\mathrm{u}$ and $\mathrm{w}$ and the temperature $\theta$ at the centre of the channel respectively for various values of the Hartmann parameter $\mathrm{M}$ and for $\tau_{D}=0,0.05$ and 0.1 . In these figures $\mathrm{m}=1$ and $\mathrm{S}=1$. Both the velocity components $\mathrm{u}$ and $\mathrm{w}$ and the temperature $\theta$ reduces with increase the intensity of the magnetic field throughout the fluid region. The similar behaviour has observed in the figures (7-9) with increase in the inverse Darcy parameter $\mathrm{D}^{-1}$. Lower the permeability of the porous medium lesser the fluid speed as observed in the entire fluid region. Figures (10-12) depict the variation of the velocity components u and w and the temperature $\theta$ at the centre of the channel $(\mathrm{y}=0)$ with time respectively for various values of the hall parameter $\mathrm{m}$ and for $\tau_{D}=0.05$. In these figures $\mathrm{M}=2$ and $\mathrm{S}=1$. Fig. 10 shows that $\mathrm{u}$ increases with increasing $\mathrm{m}$ as the effective conductivity $\left(\frac{\sigma}{1+m^{2}}\right)$ decreases with increasing $\mathrm{m}$ which reduces the magnetic damping force on $\mathrm{u}$. It is observed also from the figure that the time at which $\mathrm{u}$ reaches its steady state value increases with increasing $\mathrm{m}$ while it decreases when $\tau_{D}$ increases. The effect of $\tau_{D}$ on $\mathrm{u}$ becomes more pronounced for large values of $\mathrm{m}$. In Fig. 11 the velocity component $\mathrm{w}$ increases with increasing $\mathrm{m}$ as $\mathrm{w}$ is a result of the Hall effect. On the other hand, at small times, w decreases when $m$ increases. This happens due to the fact that, at small times $\mathrm{w}$ is very small and then the source term of $\mathrm{w}$ is proportional to $\left(\frac{m u}{1+m^{2}}\right)$ which decreases with increasing $\mathrm{m}(\mathrm{m}>1)$. Figs. 10 and 11 indicate also that the influence of $\tau_{D}$ on $\mathrm{u}$ and w depends on $\mathrm{m}$ and becomes more clear when is $\mathrm{m}$ large. An interesting phenomenon is observed in Figs. 10 and 11, which is that, when $\mathrm{m}$ has a non-zero value the component $u$ and, sometimes, $w$ overshoot. For some times they exceed their steady state values and then go down towards steady state. This may be explained by stating that with the progress of time, $u$ increases and consequently $w$ increases according to equation (4.2.14) until $\mathrm{w}$ reaches its maximum value. The increase in $\mathrm{w}$ results in a small decrease in $\mathrm{u}$ according to equation (4.2.13). This reduction in u may, in turn, result in a decrease in w according to equation (4.2.14) which explains the reduction after the peaks. The time at which overshooting occurs decreases with increasing $\tau_{D}$. Fig. (12 and 18) 
shows that the influence of $\mathrm{m}$ on $\theta$ depends on t. Increasing $\mathrm{m}$ decreases $\theta$ at small times and increases it at large times. This is due to the fact that, for small times, $u$ and $w$ are small and an increase in $m$ increases $u$ but decreases w. Then, the Joule dissipation which is also proportional to $\left(\frac{1}{1+m^{2}}\right)$ decreases. For large times, increasing $\mathrm{m}$ increases both $\mathrm{u}$ and $\mathrm{w}$ and, in turn, increases the Joule and viscous dissipations. This accounts for the crossing of the curves of $\theta$ with time for all values of $\tau_{D}$. It is also observed that increasing $\tau_{D}$ decreases the temperature $\theta$ for all values of $\mathrm{m}$. This is because increasing $\tau_{D}$ decreases both $\mathrm{u}$ and $\mathrm{w}$ and their gradients which decrease the Joule and viscous dissipations. The figure shows also that the time at which $\theta$ reaches its steady state value increases with increasing $\mathrm{m}$ while it is not greatly affected by changing $\tau_{D}$. Figures (13-15) show the effect of the suction parameter $\mathrm{S}$ on the development of the velocity components $\mathrm{u}$ and $\mathrm{w}$ and the temperature $\theta$ at $\mathrm{y}=0$ with time respectively for various values for $\tau_{D}=0.05$. In these figures $\mathrm{M}=2$ and $\mathrm{m}=1$. Figure (13) shows that $\mathrm{u}$ at the centre of the channel decreases with increasing $\mathrm{S}$ for all values of $\tau_{D}$ due to the convection of the fluid from regions in the lower half to the centre, which has higher fluid speed. Figure (14) shows that $\mathrm{w}$ increases with increasing $\mathrm{S}$ for all fixed values of governing parameters as a result of decreasing $\mathrm{u}$ which affects the source term of $\mathrm{w}$. The figure presents also the influence of $\mathrm{S}$ on the reduction of the overshooting in w especially for small values of $\tau_{D}$. Figure (15) indicates that increasing $\mathrm{S}$ decreases the temperature at the centre of the channel for all fixed values. This is due to the influence of the convection in pumping the fluid from the cold lower half towards the centre of the channel. Figures (16-18) represent the time development of the velocity components $\mathrm{u}, \mathrm{w}$ and the temperature $\theta$. Both the velocity components $\mathrm{u}$ and $\mathrm{w}$ and the temperature $\theta$ increases with all fixed values of governing parameters throughout the fluid region. The tables (I-II) represent the Nusselt number on $y=1$ and $y=-1$ levels. We notice that the rate of heat transfer increases with increase in $\tau_{D}, \mathrm{M}, \mathrm{m}$ and $\mathrm{D}^{-1}$, decreases with increases in $\mathrm{S}$ at $y=1$ level. The similar behaviour is observed at $y=-1$ level.

\section{Conclusions}

The transient Couette flow of a Bingham non-Newtonian fluid under the influence of an applied uniform magnetic field with the normal to the boundaries in the transverse xz-plane is studied considering the hall effect. The effects of the Bingham number $\tau_{D}$, the Hartmann number $\mathrm{M}$, the inverse Darcy parameter $\mathrm{D}^{-1}$, the Hall parameter $\mathrm{m}$, and the suction parameter $\mathrm{S}$ on the velocity and temperature distributions are studied.

1. The hall term affect the main velocity component $\mathrm{u}$ in the $\mathrm{x}$-direction and gives rise to another velocity component $\mathrm{w}$ in the $\mathrm{z}$-direction. An overshooting in the velocity components $\mathrm{u}$ and $\mathrm{w}$ with time due to the Hall effect is observed for fixed value of $\tau_{D}$.

2. The flow index $\tau_{D}$ has an apparent effect in controlling the overshooting in $\mathrm{u}$ or $\mathrm{w}$ and the time at which it occurs.

3. The results show that the influence of the parameter $\tau_{D}$ on $u$ and $w$ depends on $\mathrm{m}$ and becomes more apparent when $\mathrm{m}$ is large.

4. It is found also that the effect of $\mathrm{m}$ on $\mathrm{w}$ depends on $\mathrm{t}$ for all values of $\tau_{D}$ which accounts for a crossover in the $\mathrm{w}-\mathrm{t}$ graph for various values of $\mathrm{m}$.

5. The effect of $\mathrm{m}$ on the magnitude of $\theta$ depends on $\mathrm{n}$ and becomes more pronounced in case of small $\tau_{D}$.

6. The time at which $\mathrm{u}$ and $\mathrm{w}$ reach the steady state increases with increasing $\mathrm{m}$, but decreases when $\tau_{D}$ increases.

7. The time at which $\theta$ reaches its steady state increases with increasing $\mathrm{m}$ while it is not greatly affected by changing $\tau_{D}$.

8. The magnitude of the velocity components $\mathrm{u}$, w and $\theta$ reduces with increases $\mathrm{M}$ and $\mathrm{D}^{-1}$.

9. Lower the permeability of the porous medium lesser the fluid speed as observed in the entire fluid region. 
10. The rate of heat transfer increases with increase in $\tau_{D}, \mathrm{M}, \mathrm{m}$ and $\mathrm{D}^{-1}$, decreases with increases in $\mathrm{S}$ at $y=1$ and $y=-1$ levels.

\section{References}

[1]. Abo-El-Dahab. E.M.H., "Effect of Hall Currents on Some Magneto hydro dynamic Flow Problems", Master's Thesis, Department of Maths, Faculty of Science, Helwan University, Egypt, 1993.

[2]. Alpher. R.A., "Heat transfer in magneto hydro dynamic flow between parallel plates", International Journal of Heat Mass Transfer, Vol. 3 (1961), p. 108.

[3]. Antia. M., "Numerical Methods for Scientists and Engineers", Tata McGraw-Hill, New Delhi, 1991.

[4]. Bird. R.B., G.C. Dai, B.J. Yarusso, "The rheology and flow of visco-plastic materials", Review of Chemical Engineering., Vol. 1 (1983), pp. 36-69.

[5]. Chamkha. A.J., "Hydro magnetic two-phase flow in a channel", International Journal of Engineering Science, Vol. 33 (3) (1995), pp. $437-446$.

[6]. Chamkha. A.J., "Unsteady flow of an electrically conducting dusty-gas in a channel due to an oscillating pressure gradient", Applied Mathematical Modelling, Vol. 21 (1997), pp. 287-292.

[7]. Chamkha. A.J., "Unsteady laminar hydromagnetic fluid-particle flow and heat transfer in channels and circular pipes", International Journal of Heat Fluid Flow, Vol. 21 (2000), pp. 740-746.

[8]. Hazem Ali Attia and M.E.Sayed Ahmed., "Hall effects on unsteady MHD coquette flow and heat transfer of a Bingham fluid with suction and injection", Applied Mathematical Modelling, Vol. 28 (2004), pp.1027-1045.

[9]. Hazem Ali Attia and N.A. Kotb, “MHD flow between two parallel plates with heat transfer”, Acta Mechanica, Vol. 117 (1996), pp. $215-220$.

[10]. Hazem Ali Attia, "Hall current effects on the velocity and temperature fields of an unsteady Hartmann flow", Canadian Journal of Physics, Vol. 76 (9) (1998), pp. 739-746.

[11]. Hazem Ali Attia, "Transient, MHD flow and heat transfer between two parallel plates with temperature dependent viscosity", Mech. Res. Comm. Vol. 26 (1) (1999), pp. 115-121.

[12]. Hazem Ali Attia, "Unsteady MHD flow and heat transfer of dusty fluid between parallel plates with variable physical properties", Applied Mathematical Modelling, 13 (9) (2002), pp. 863-875.

[13]. Hazem Ali Attia, "The effect of variable properties on the unsteady Hartmann flow with heat transfer considering the Hall effect", Applied Mathematical Modelling, Vol. 27 (7) (2003), pp. 551-563.

[14]. Min. T., J.Y. Yoo and H. Choi, "Laminar convective heat transfer of a Bingham plastic in a circular pipe. II. Numerical approach hydro dynamically developing flow and simultaneously developing flow, International Journal of Heat Mass Transfer, Vol. 40 (15) (1997), pp. 3689-3701.

[15]. Nigam S.D. and S.N. Singh, "Heat transfer by laminar flow between parallel plates under the action of transverse magnetic field", Quarterly Journal of Mechanics and Applied Mathematics, Vol. 13 (1960), p. 85.

[16]. Patel. N. and D.B. Ingham, "Mixed convection flow of a Bingham plastic in an eccentric annulus", International Journal of Heat Flow, Vol. 15 (2) (1994), pp. 132-141.

[17]. Petrov. A.G., "The development of the flow of viscous and visco plastic media between two parallel plates", Journal of Applied Mathematics and Mechanics. Vol. 64 (1) (2000), pp. 123-132.

[18]. Schlichting. H., "Boundary Layer Theory", McGraw-Hill, New York, 1986.

[19]. Soundalgekar. V.M. and N.V. Vighnesam, H.S. Takhar, "Hall and ion-slip effects in MHD Couette flow with heat transfer", IEEE Trans. Plasma Sci. PS-7 (3 September) (1979), pp. 178-182.

[20]. Soundalgekar. V.M., A.G. Uplekar, "Hall effects in MHD Couette flow with heat transfer", IEEE Trans. Plasma Sci. PS-14 (5 October) (1986), pp. 579-583.

[21]. Sutton G.W. and A. Sherman, "Engineering Magneto hydro dynamics", McGraw-Hill, New York, 1965.

[22]. Tani. I., "Steady motion of conducting fluids in channels under transverse magnetic fields with consideration of Hall effect", Journal of Aerospace Science, Vol. 29 (1962) 287.

[23]. Tao. I.N., "Magneto hydro dynamic effects on the formation of Couette flow", Journal of Aerospace Science, Vol. 27 (1960), p. 334.

[24]. Vradis. G.C., J. Dougher, S. Kumar, "Entrance pipe flow and heat transfer for a Bingham plastics", International Journal of Heat Mass transfer, Vol. 96 (1993), pp. 543-550.

[25]. Walton. I.C. and S.H. Bittleston, "The axial flow of a Bingham plastic in a narrow eccentric annulus", Journal of Fluid Mechanics, Vol. 222 (1991), pp. 39-60. 